\title{
AÁREA NOROESTE DA BACIA DE CAMPOS, BRASIL, DO MESOCRETÁCEO AO NEOCRETÁCEO: EVOLUÇÃO PALEOAMBIENTAL E PALEOGEOGRÁFICA PELO ESTUDO DE FORAMINÍFEROS
}

\author{
EDUARDO A.M. KOUTSOUKOS*
}

\begin{abstract}
Foraminifaral studies carried out in 15 exploratory wells, drilled offshore by Petrobrás in the northern portion of Campos Basin, São Tomé Low area, allowed the reconstruction of the regional biochronostratigraphic framework and paleogeographic evolution from "Mid" to Late Cretaceous. Correlative well sections and paleoenvironmental maps are presented, and the following main conclusions are drawn: $a$ ) continous subsidence with progressive bathymetric increment controlled the deposition in the area from the Albian to Maastrichtian; $b$ ) the deepening process was strongly increased during the Late Cretaceous with the establishment of an oceanic depositional setting; $c$ ) the "Mid" Cretaceous was characterized by relatively stable sedimentation in neritic environment; $d$ ) restrictive paleoceanographic conditions (low oxygen content) were widespread in the Cenomanian/ Early Turonian (?), with a scarce and low-diversity microfauna; $e$ ) the present configuration of the São Tomé Low can be well recognized since the Maastrichtian, the apparent direction of its depositional trend being SW-NE.
\end{abstract}

INTRODUÇÃo Os indícios de hidrocarbonetos presentes no pacote sedimentar cretácico da porção noroeste da Bacia de Campos, até certo ponto expressivos, motivaram a realização deste estudo, cujos resultados preliminares já foram apresentados pelo autor (Koutsoukos 1984). Como objetivos principais, buscou-se para a área:

- conhecer com detalhe a seqüência biocronoestratigráfica; - caracterizar o arranjo de paleoambientes deposicionais ao longo das seções;

- elaborar um modelo de evolução paleoambiental e paleogeográfica.

O grupo de poços estudados e sua localização são apresentados na figura 1 . Os resultados referentes às seções $A-A$, e $D-D$ ' (evolução paleobatimétrica e relação biota/habitat através do tempo), bem como o contexto geológico e investigações anteriores realizadas nesta área encontram-se em Koutsoukos (1984).

MÉTODOS DE TRABALHO Os métodos e critérios utilizados para os estudos biocronoestratigráficos e paleoecológicos aqui empregados estão apresentados em detalhe em Koutsoukos (1984).

Foram analisadas 394 amostras de calha e 40 de testemunhos, coletadas seletivamente de 15 poços exploratórios submarinos, tomando-se, de preferência, sedimentos de granulometria fina.

A interpretação paleoecológica baseou-se na composição taxonômica, diversidade e freçüência de comunidades de foraminíferos (Fig. 2). A freqüência relativa de outros organismos associados, as condições gerais de preservação das tecas e características litológicas constituíram-se, também, em importantes parâmetros para a reconstituição paleoambiental.

BIOESTRATIGRAFIA O arcabouço biocronoestratigráfico empregado neste trabalho teve por base o detalhamento e a integração dos zoneamentos desenvolvidos para a margem continental brasileira pela Petrobrás (Fig. 3). Sub-

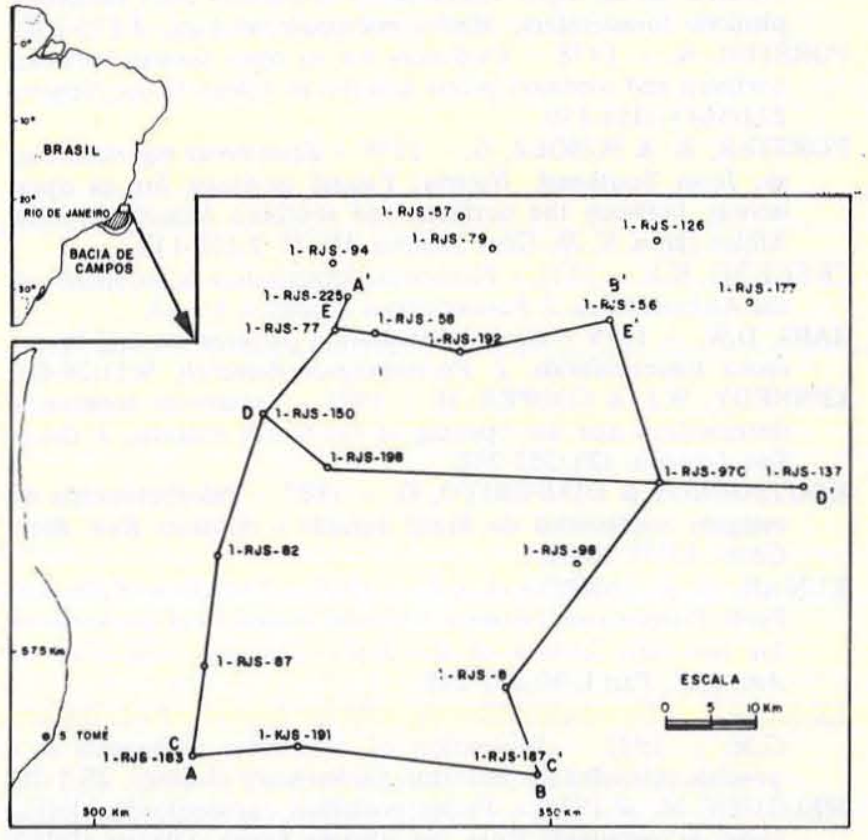

Figura 1-Mapa de situação dos poços das seções estudadas

dividiram-se informalmente, ajustando-se à escala padrão de tempo geológico, as seguintes biozonas de foraminíferos:

Zona Favusella washitensis, parte inferior: atribuída ao Eo-Mesoalbiano.

Zona Favusella washitensis, parte superior: Neoalbiano.

Zona Hedbergella delrioensis, parte inferior: Cenomaniano-Eoturoniano (?).

Zona $H$. delrioensis, parte média: aproximadamente Coniaciano.

Zona $H$. delrioensis, parte superior: Santoniano.

As associações de microfósseis diagnósticos das referidas unidades estão listadas na figura 3. 

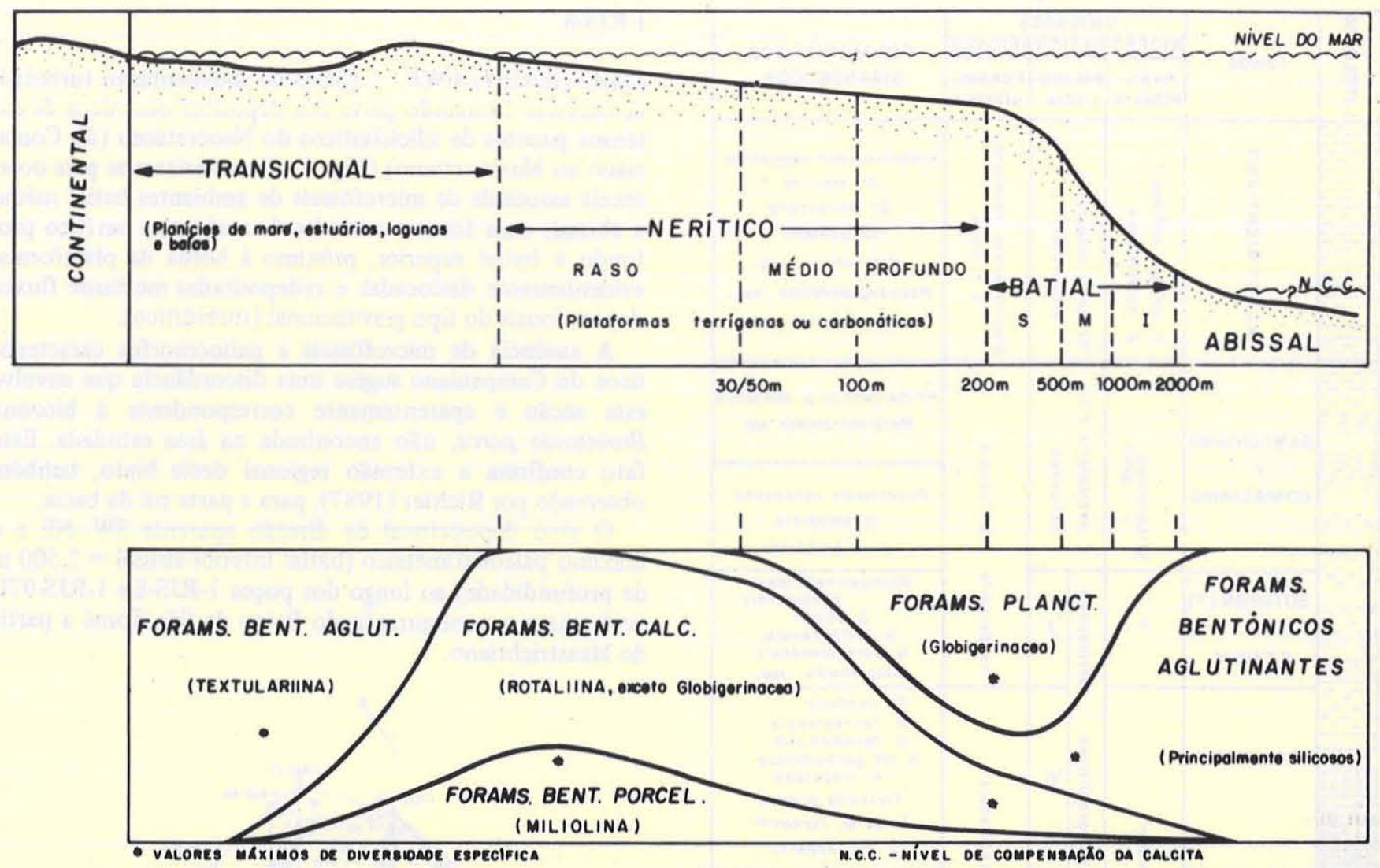

Figura 2 - Modelo esquemático dos ambientes marinhos. Distribuição batimétrica e abundância relativa dos principais grupos de foraminiferos (adaptado de Koutsoukos \& Merrick 1985)

DISCUSSÃo PALEOECOLÓgICA As análises do conteúdo de foraminíferos permitiram a construção de seções correlativas concernentes à cronoestratigrafia e às unidades paleoecológicas reconhecidas, reunidas no diagrama emcerca da figura 4. Mapas paleobatimétricos, estabelecidos em topos de horizontes biocronoestratigráficos e elaborados com base nas indicações paleobatimétricas fornecidas pelas associações de foraminíferos, são apresentados na figura 5.

A situação no Mesocretáceo EO-MESOALBIANO Esta , fase caracterizou-se por um registro sedimentar de clásticosarenosos intercalados com calcarenitos, gradando a calcilutitos para leste, depositado em ambiente nerítico raso-médio, decorrente de uma transgressão marinha sobre uma plataforma extensa e de fundo relativamente plano (Fig. 5).

A interpretação paleoecológica sugere uma faixa ligeiramente mais profunda em direção à borda da bacia, no final desta fase. Esta variação seria, na realidade, muito pequena (da ordem de 10 a $20 \mathrm{~m}$ ) e seu conteúdo biótico, representado por freqüêntes foraminíferos planctônicos (Favusella spp., Globigerinelloides ex gr. caseyi, Hedbergella spp., Heterohelix aff. moremani e Ticinella spp.), e uma fauna bentônica pouco diversificada, que indicam uma faixa de circulação mais livre, menos restrita.

NEOALBIANO Neste tempo deu-se a sedimentação de margas, gradando a calcilutitos para leste, sob condições de nerítico médio-profundo (média de $\approx 100 \mathrm{~m}$ de profundidade), na maior parte da área (Fig. 5). $\mathrm{O}$ conteúdo fossilífe- ro é caracterizado por abundantes foraminíferos planctônicos (Globigerinelloides spp., Hedbergella ssp., Rotalipora sp., Ticinella spp. e Whiteinella spp.) e maior diversidade e freqüência de formas bentônicas.

A sudoeste, entretanto, na área do poço 1-RJS-87, acumularam-se depósitos em ambiente nerítico raso-médio ( $\approx$ 30 a $50 \mathrm{~m}$ de profundidade), com uma biota pouco freqüênte e de baixa diversidade. Já na área dos poços 1-RJS-77 e 1-RJS-58, a presença de oólitos e microgastrópodes poderia sugerir que, ainda durante esta fase, bancos carbonáticos rasos ocorriam localmente nas imediações.

CENOMANIANIO/EOTURONIANO (?) Nesta fase, a área encontrava-se sob condições paleobatimétricas em torno de $150 \mathrm{~m}$ (nerítico profundo), com a acumulação de margas e folhelhos contendo uma biota escassa e de baixa diversidade, em franco contraste com a abundância faunística da fase anterior (Fig. 5). Essas características estariam refletindo uma situação anômala ligada a um processo de anoxia que parece ter sido generalizado no Atlântico Sul durante este tempo (Ryan \& Cita 1977, Arthur \& Schalanger 1979, Graciansky et al. 1984, Azevedo et al. 1987, Dias-Brito 1982, Viviers \& Regali 1987).

A ausência de depósitos pertencentes a esta fase nos poços 1-RJS-56, 1-RJS-77, 1-RJS-97C, 1-RJS-137, 1-RJS-150, 1-RJS-192 e 1-RJS-225 e em vários outros sítios da margem continental atlântica (Koutsoukos \& Merrick 1986) estaria relacionada com a ocorrência de um evento erosivo ou nãodeposicional de grande escala que marcou o Atlântico Sul 


\begin{tabular}{|c|c|c|c|c|c|c|}
\hline \multirow{2}{*}{\begin{tabular}{|l|}
5 \\
0 \\
0 \\
0 \\
0 \\
\\
\end{tabular}} & \multirow{2}{*}{ IDADE } & \multicolumn{4}{|c|}{$\begin{array}{c}\text { UNIDADES } \\
\text { BIOESTRATIGRÁFICAS * }\end{array}$} & \multirow{2}{*}{$\begin{array}{l}\text { FORAMINÍFEROS } \\
\text { DIAGNÓSTICOS }\end{array}$} \\
\hline & & $\begin{array}{l}\text { NANO- } \\
\text { FÓSSEIS }\end{array}$ & $\begin{array}{l}\text { PALI } \\
\text { LOG }\end{array}$ & & $\begin{array}{l}\text { FORAMI- } \\
\text { NÍFEROS }\end{array}$ & \\
\hline & 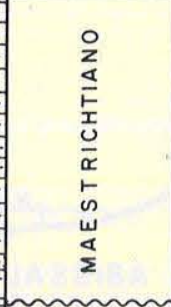 & 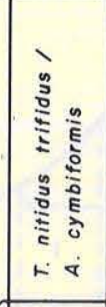 & $\begin{array}{l}0 \\
\vdots \\
\vdots \\
\vdots \\
\vdots \\
0 \\
0 \\
\vdots \\
\vdots \\
\vdots \\
0 \\
0 \\
0 \\
0\end{array}$ & & 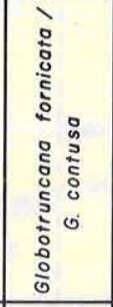 & $\begin{array}{c}\text { Globotruncana aegyptiaca } \\
\text { Gt. olevata } \\
\text { Gt. fornicata } \\
\text { Gt. gansseri } \\
\text { Heterohelix spp. } \\
\text { Pseudoguembelina spp. } \\
\text { Rugoglobigerina rugosa }\end{array}$ \\
\hline & SANTONIANO & $n \leqslant$ & $\begin{array}{l}n \\
\vdots \\
\vdots \\
\vdots \\
\vdots\end{array}$ & $\frac{n}{n}$ & 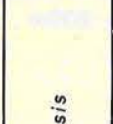 & $\begin{array}{l}\text { Hedbergella ex. gr. delrioensis } \\
\text { Marginotruncano spp. }\end{array}$ \\
\hline & CONIACIANO & 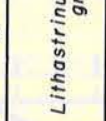 & $\mid \begin{array}{c}3 \\
\vdots \\
0 \\
\vdots \\
\vdots \\
0 \\
0\end{array}$ & 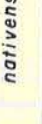 & $\begin{array}{l}\vdots \\
\vdots \\
\vdots \\
\vdots\end{array}$ & $\begin{array}{c}\text { Dicarinella concavata } \\
\text { H. amabillis } \\
\text { H. planispira }\end{array}$ \\
\hline$\overline{-}$ & $\begin{array}{c}\text { EOTURON. (?) } \\
/ \\
\text { CENOM. }\end{array}$ & $?$ & 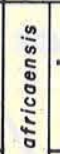 & $" \gamma "$ & 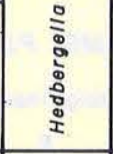 & $\begin{array}{c}\text { Globigerinelloldes } \\
\text { bentonensis } \\
\text { G. caseyi } \\
\text { H. borittonensis } \\
\text { H. Portsdownensis } \\
\text { Whiteinella spp. }\end{array}$ \\
\hline 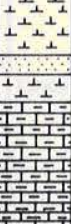 & NEOALBIANO & 亲 & 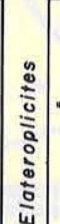 & $" \beta "$ & $\begin{array}{l}\frac{5}{5} \\
\vdots \\
\vdots \\
\vdots \\
\vdots \\
\vdots\end{array}$ & $\begin{array}{l}\text { F. confusa } \\
\text { G. ferreolensis } \\
\text { G. texomaensis } \\
\text { H. aff. gorbachikae } \\
\text { H. trocoidea } \\
\text { Ticinella primula } \\
\text { T. ex. gr. raynoudi } \\
\text { T. aff. roberti }\end{array}$ \\
\hline 4 & $\begin{array}{c}\text { MESOALBIANO } \\
\text { EOALBIANO }\end{array}$ & 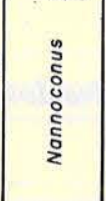 & $\begin{array}{c}1 \\
\vdots \\
\vdots \\
\vdots \\
\vdots \\
\vdots \\
\vdots \\
\vdots \\
\vdots \\
0 \\
0 \\
0\end{array}$ & $" \alpha "$ & 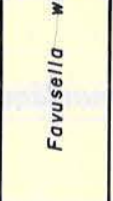 & $\begin{array}{l}\text { Favusella confusa } \\
\text { F. washitensis } \\
\text { H. aff. gorbachikae } \\
\text { H. aff. trocoidea } \\
\text { Trocholina sp. }\end{array}$ \\
\hline
\end{tabular}

* Segundo zoneamentos da Petrobrás (Troelsen \& Quadros 1971, Regali et al. 1974, Uesugui 1976, Noguti \& Santos 1975, modificado)

Figura 3 - Correlação biocronoestratigráfica das unidades observadas na área estudada

neste tempo. Butt (1982), Koutsoukos \& Merrick (op. cit.) e Dias-Brito (1987) consideram que a ausência de tais registros poderiam estar ligada à desestabilização do Atlântico Sul, em decorrência de sua conexão efetiva com o Atlântico Norte (Tethys), ocorrida entre o Cenomaniano e o Turoniano.

\section{A Situação do Neocretáceo CONIACIANO-SANTO-} NIANO 0 eixo deposicional de direção aparente SW-NE começa, a partir desta fase, a se estabelecer com um registro sedimentar de soliclásticos pelíticos (Fig. 5).

A microfauna é composta de foraminíferos bentônicos bastante diversificados, com predominância de formas aglutinantes, abundantes radiolários e escassos foraminíferos planctônicos (Dicarinella ex gr. concavata, Globotruncana spp., Hedbergella spp. e Marginotruncana spp.).

Os depósitos de ambiente nerítico profundo-batial superior restringem-se à porção proximal da bacia, ainda não sendo claramente definida a borda da plataforma, que deveria situar-se nas imediações do limite apresentado na figura 5. Por outro lado, os sedimentos mais profundos, de ambiente batial médio-inferior, encontram-se na área do poço
1-RJS-8.

MAASTRICHTIANO Ciclos de sedimentação turbidítica ocorrem formando parte dos depósitos oceânicos de extensos pacotes de siliciclásticos do Neocretáceo (do Coniaciano ao Maastrictiano) (Fig. 5). Caracterizam-se pela ocorrência associada de microfósseis de ambientes batial médio a abissal, com formas oriundas de ambientes nerítico profundo a batial superior, próximo à borda da plataforma, evidentemente deslocadas e redepositadas mediante fluxos deposicionais do tipo gravitacional (turbidítico).

A ausência de microfósseis e palinomorfos característicos do Campaniano sugere uma discordância que envolve esta seção e aparentemente correspondente à biozona Broinsonia parca, não encontrada na área estudada. Este fato confirma a extensão regional deste hiato, também observado por Richter (1987), para a parte sul da bacia.

$\mathrm{O}$ eixo deposicional de direção aparente $\mathrm{SW}-\mathrm{NE}$ e o máximo paleobatimétrico (batial inferior-abissal $\approx 2.500 \mathrm{~m}$ de profundidade) ao longo dos poços 1-RJS-8 e 1-RJS-97C evidenciam a configuração do Baixo de São Tomé a partir do Maastrichtiano.

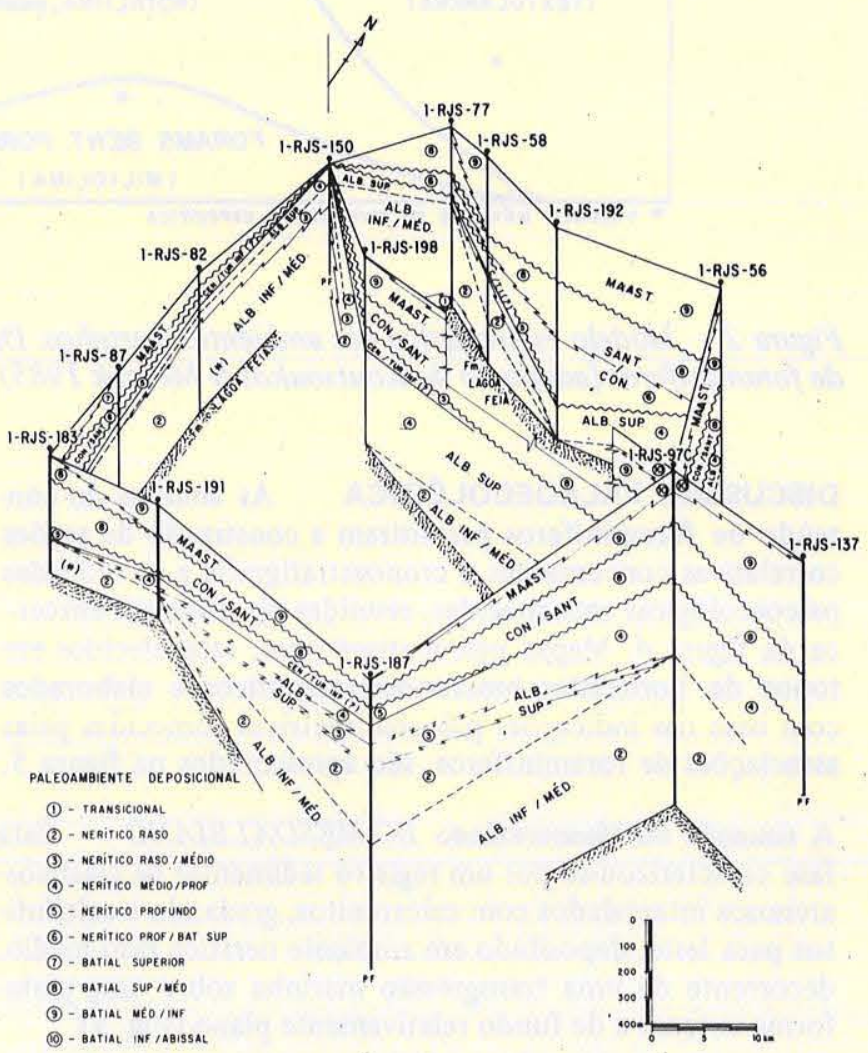

Figura 4 - Diagrama em cerca. Fácies arenosa da Formação de Macaé (segundo Guardado et al. 1983)

\section{CONCLUSÕES}

1. Do Albiano ao Cenomaniano-Eoturoniano (?) esta área da bacia manteve-se sob relativa estabilidade paleobatimétrica, com a deposição dos sedimentos tendo ocorrido em meio nerítico.

2. A partir do Coniaciano, o processo de progressivo aumento paleobatimétrico foi acentuadamente intensificado, iniciando-se, então, o acúmulo de sedimentos em condições 


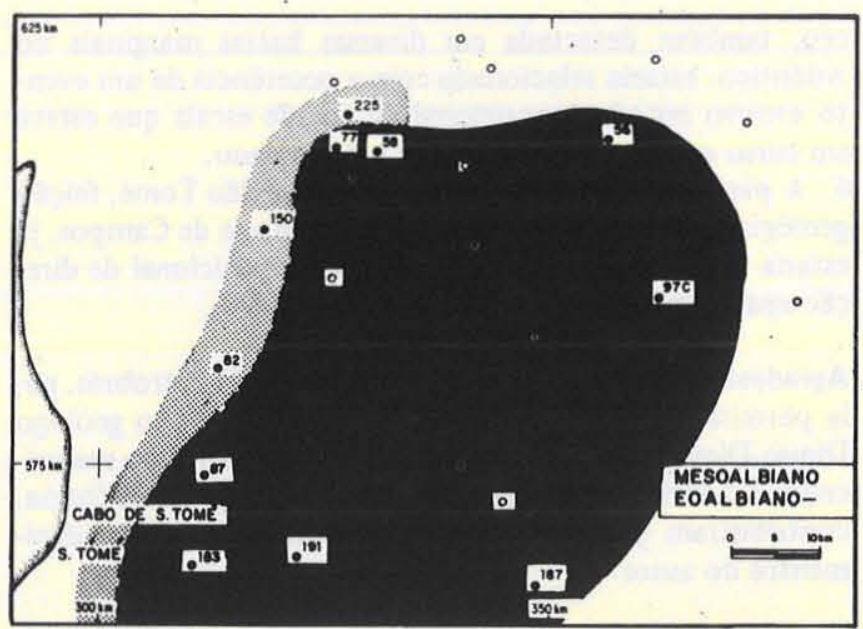

- POCOS DE CONTROLE
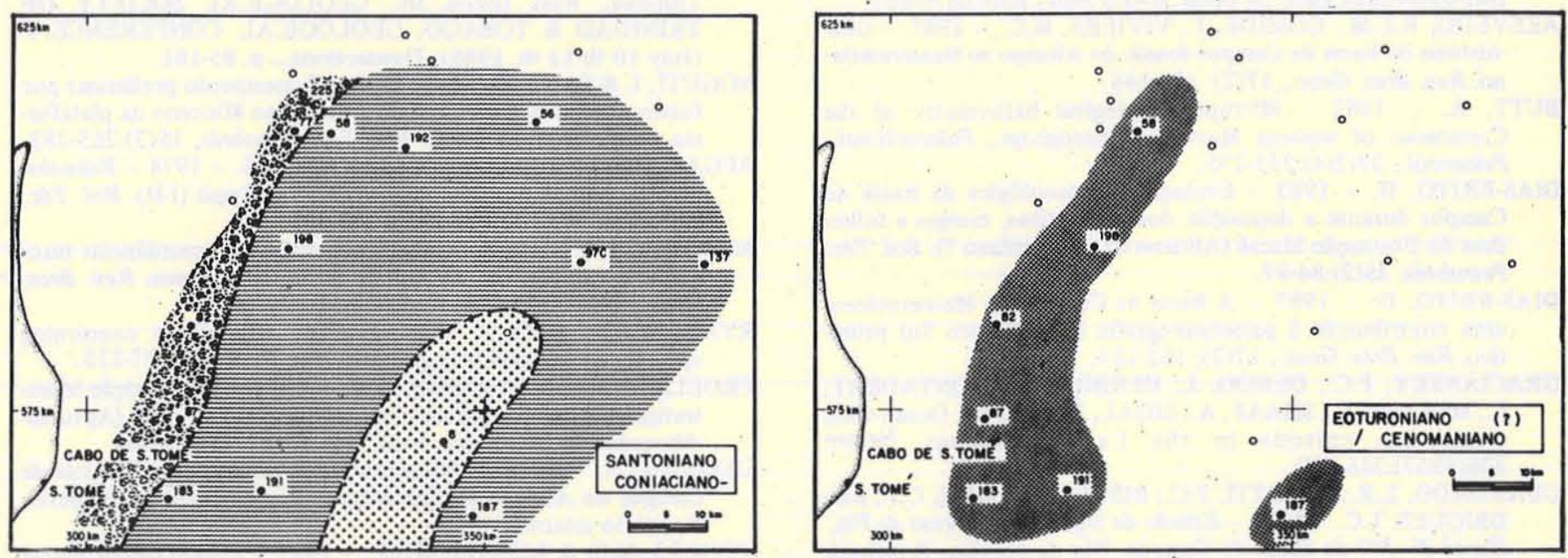

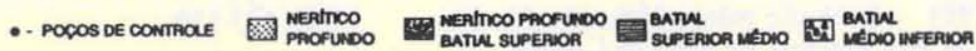

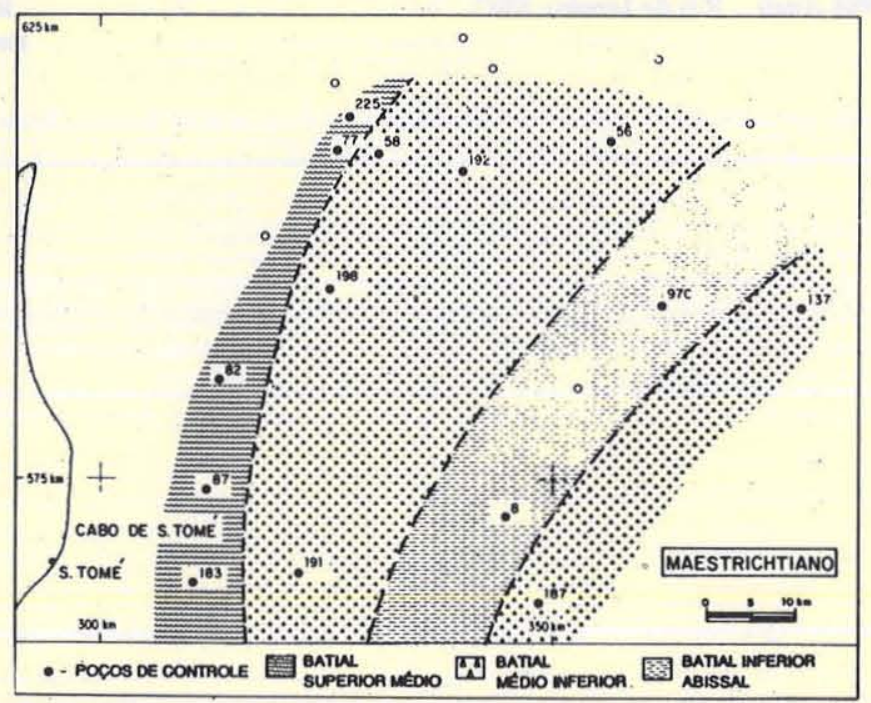

Figura 5-Mapas paleobatimétricos 
oceânicas. Tal incremento prolonga-se através de todo o Neocretáceo.

3. Durante todo o Albiano, o depocentro da área estava situado a SE; a fácies arenosa da Formação Macaé, depositada no Eo-Mesoalbiano, acumulou-se em ambiente nerítico raso, proximal-costeiro.

4. O intervalo Cenomaniano/Eoturoniano (? ), caracterizado por uma microfauna escassa e de baixa diversidade, foi marcado por condições oceanográficas especiais, em que folhelhos e margas teriam sido depositados sob condições de anoxia.

5. A ausência de depósitos do Cenomaniano-Eoturoniano (? ), em vários poços da área estudada e do Campaniano, em sua totalidade, revela a existência de dois consideráveis hiatos nesta porção da bacia. A descontinuidade do Mesocretá- ceo, também detectada em diversas bacias marginais do Atlântico, estaria relacionada com a ocorrência de um evento erosivo ou não-deposicional de grande escala que esteve em curso entre o Cenomaniano e o Turoniano.

6. A partir do Maastrichtiano, o Baixo de São Tomé, feição geológica proeminente nesta porção da Bacia de Campos, já estaria bem configurado, com seu eixo deposicional de direção aparente SW-NE.

Agradecimentos À diretoria executiva da Petrobrás, pela permissão para a apresentação e publicação; ao geólogo Dimas Dias-Brito, pelas sugestões e leitura crítica do manuscrito; e a todos os colegas que, de uma ou de outra forma, contribuíram para a realização deste trabalho, os agradecimentos do autor.

\section{REFERÊNCIAS BIBLIOGRÁFICAS}

ARTHUR, M.A. \& SCHALANGER, S.O. - 1979 - Cretaceous "oceanic anoxic events" as causal factors in development of reef-reservoired giant oil fields. AAPG Bull., 63(6):870-885.

AZEVEDO, R.L.M.; GOMIDE, J.; VIVIERS, M.C. - 1987 - Geo-história da Bacia de Campos-Brasil: do Albiano ao Maastrichtiano. Rev. Bras. Geoc., 17(2): 139-146.

BUTT, A. - 1982 - Micropaleontological bathymetry of the Cretaceous of western Morocco. Palaeogeogr., Palaeoclimat., Palaeocol., 37(2/4):235-275.

DIAS-BRITO, D. - 1982 - Evolução paleoecológica da Bacia de Campos durante a deposição dos calcilutitos, margas e folhelhos da Formação Macaé (Albiano e Cenomaniano ?). Bol. Téc. Petrobrás, 25(2):84-97.

DIAS-BRITO, D. - 1987 - A Bacia de Campos no Mesocretáceo: uma contribuição à paleoceanografia do Atlântico Sul primitivo. Rev. Bras. Geoc., 17(2): 162-167.

GRACIANSKY, P.C.; DEROO, J.; HERBIL, J.P.; MONTADERT, L.; MULLER, C.; SHAAF, A.; SIGAL, J. - 1984 - Ocean-wide stagnation episode in the Late Cretaceous. Nature 308(5957):346-349.

GUARDADO, L.R.; COLETTI, P.C.; BISOL, D.L.; APPI, C.J.; RODRIGUES, T.C. - 1983 - Estudo da seção de água rasa da Fm. Macaé N-NO da Bacia de Campos. Rio de Janeiro, Petrobrás, (Rel. int.).

KOUTSOUKOS, E.A.M. - 1984 - Evolução paleocológica do Albiano ao Maastrichtiano na área noroeste da Bacia de Campos, Brasil, com base em foraminíferos. In: CONGR. BRAS. GEOL., 33, Rio de Janeiro, 1984 Anais... Rio de Janeiro, SBG. v. 2, p. 685-698.
KOUTSOUKOS, E.A. M \& MERRICK, K.A. - 1986 - Foraminiferal paleoenvironments from the Barremian to Maastrictian of Trinidad, West Indies. In: GEOLOGICAL SOCIETY OF TRINIDAD \& TOBAGO, GEOLOGICAL CONFERENCE, 1 (July 10 th-12 th, 1985). Transactions... p. 85-101.

NOGUTI, I. \& SANTOS, J.F. - 1972 - Zoneamento preliminar por foraminíferos planctônicos do Aptiano ao Mioceno na plataforma continental do Brasil. Bol. Téc. Petrobrás, 15(3):265-283.

REGALI, M.S.P.; UESUGUI, N.; SANTOS, A.S. - 1974 - Palinologia dos sedimentos Meso-Cenozóico do Brasil (I-II). Bol. Téc. Petrobrás, 17:(3-4):177-191 e 263-301.

RICHTER, A.J. - 1987 - Subafloramento das discordâncias turoniana e campaniana no sul da Bacia de Campos. Rev. Bras. Geoc., 17(2): 173-176.

RYAN, W.B.E. \& CITA, M.B. - 1977 - Ignorance concerning episodes of oceanwide stagnation. Mar. Geol., 23:197-215.

TROELSEN, J.C. \& QUADROS, L.P. - 1971 - Distribuição bioestratigráfica dos manofósseis em sedimentos marinhos (Aptiano-Mioceno) do Brasil. An. Acad. bras. Ciên. 43:577-609.

UESUGUI, N. - 1976 - Intervalos bioestratigráficos da Bacia de Campos do Albiano ao Santoniano. Rio de Janeiro, Petrobrás, Relatório interno.

VIVIERS, M.C. \& REGALI, M.S.P. - 1987 - Estudo paleoambiental preliminar no Cretáceo da Bacia Potiguar. Rev. Bras. Geoc. 17(2): 123-130.

MANUSCRITO 385

Recebido em 01 de Setembro de 1986 Revisão aceita em 30 de Março de 1987

"O berilo (silicato) que exportamos a baixo preço, estabelecido alhures, obtém uma valorização de 35 vezes, se em vez de silicato, exportássemos o óxido de berilo ( $\mathrm{BeO}$ ) ou se, em vez do berilo-gema (água marinha) bruta a exportássemos lapidada". 